\title{
Internal shocks and the blazar sequence
}

\section{Low and intermediate power BL Lac objects}

\author{
D. Guetta ${ }^{1,2}$, G. Ghisellini ${ }^{3}$, D. Lazzati ${ }^{4}$, and A. Celotti ${ }^{5}$ \\ 1 INAF - Osservatorio Astrofisico di Arcetri, Largo E. Fermi 5, 50125 Firenze, Italy \\ 2 JILA, University of Colorado, Boulder, CO 80309, USA \\ 3 INAF - Osservatorio Astronomico di Brera, via Bianchi 46, 23807 Merate, Italy \\ 4 Institute of Astronomy, Madingley Road, CB3 OHA, Cambridge, UK \\ 5 SISSA/ISAS, via Beirut 2-4, 34014 Trieste, Italy \\ Received 10 February 2004 / Accepted 21 March 2004
}

\begin{abstract}
We consider internal shocks as the main dissipation mechanism responsible for the emission in blazars and show that it can satisfactorily account for the properties of all blazars. In particular, we extend previous work (Spada et al. 2001) on powerful objects, to intermediate (BL Lac) and low power sources (Mkn 421), in order to reproduce the whole of the blazar sequence. The model self-consistently treats the dynamics, spectral emission and its variability. The key parameters driving the phenomenological sequence are the jet power and the properties of the broad line region, which regulate the cooling efficiency of the emitting particles and thus the shape of the spectral energy distribution. By assuming that the remaining parameters are similar for all objects it has been possible to reproduce the full range of the observed spectral "states". A more detailed comparison of the variability properties shows (for Mkn 421) a good agreement in the X-ray band, while in the optical the simulated flux appears to be too variable. For BL Lac lags ( $\sim 10$ days) are predicted between the $\gamma$-rays and the infrared emission.
\end{abstract}

Key words. galaxies: jets - galaxies: nuclei - radio continuum: galaxies - galaxies: individual: Mkn 421, BL Lac

\section{Introduction}

The discovery that blazars are strong $\gamma$-ray emitters together with the results of multiwavelength campaigns have allowed to deepen our knowledge on these objects. Their Spectral Energy Distribution (SED) is characterized by two broad peaks (Fossati et al. 1998) strongly variable on different timescales (Wagner \& Witzel 1995; Ulrich et al. 1997).

Two main radiation processes dominate the emission, namely synchrotron at low frequencies and - plausibly - inverse Compton at high energies (see e.g. Sikora 1994, for a review). The relative luminosity in the two peaks and their peak frequency appear to be functions of the total power (Fossati et al. 1998; Ghisellini et al. 1998), resulting into a sequence for the whole of the blazar population properties, ranging from powerful, low frequency peak, through intermediate, to low power, high frequency peak (blue) blazars (see however Padovani et al. 2003). The blazar emission is variable on energy dependent timescales, typically of weeks-months in the radio and of the order of a day in the $\gamma$-ray band.

Several studies, mainly based on the modeling of the SED, consistently derived the physical parameters of the emitting

Send offprint requests to: D. Guetta,

e-mail: dafne@arcetri.astro.it region. However key issues in the understanding of relativistic jets remain open, most notably the jet energetics and the particle acceleration process(es). In order to explore these issues and their relationship we have (quantitatively) considered a scenario in which the plasma conditions and their variability are not treated as free parameters, but follow from the jet dynamics, thus relating the observed emission properties with the energy transport along the jet. Such a scenario assumes that internal shocks are responsible for the dissipation within jets (Rees 1978; and then mostly explored for Gamma-Ray Bursts, Rees \& Mészáros 1994).

The key assumption of the model is that the energy is channeled into jets in an intermittent way by the central engine, though such a time dependent process cannot be inferred from first principles. Different parts of the jet ("shells") moving at different speeds can collide giving rise to shocks and dissipation as non-thermal radiation. The mechanism has a limited efficiency (unless the contrast in Lorentz factors between different shells is extremely large, see Beloborodov 2000; Guetta et al. 2001, but also Ghisellini 2002), which has to be indeed the case for blazars since most of the energy propagates up to the extended radio lobes.

Beside the low efficiency, the internal shock scenario can naturally account for other blazar properties. It predicts that 
jets become radiative at $\gtrsim 10^{16}-10^{17} \mathrm{~cm}$ from the central engine, implying a minimum distance from the accretion disk and a minimum dimension for the $\gamma$-ray source, as required by observations (in order to avoid copious pair production; see Ghisellini \& Madau 1996). Furthermore, successive collisions taking place at larger distances have reduced efficiency since the Lorentz factor contrast of the colliding shells decreases - explaining why the jet luminosity decreases with distance. Finally, the internal shock scenario appears a promising non steady state model to account for the observed large amplitude variability.

A detailed study of the predictions of this model via numerical simulations has been carried out by Spada et al. (2001, hereafter S01) for powerful blazars (specifically 3C 279). As the SED of this object does not represent the whole of the blazar family mentioned above, we focus here on two well studied sources representative of extremely blue and intermediate blazars, namely Mkn 421 and BL Lac itself. Rather than reproducing in detail particular spectra of specific objects, the aim of this work is to determine whether the proposed scenario, including the dynamics and emission properties, i) can account for the different SED along the blazar sequence, ii) under which hypothesis this can occur, and iii) whether the internal shock model can reproduce the observed variability behavior.

The outline of the paper is the following. In Sect. 2 we describe the hypothesis on the wind dynamics and on the radiative properties of the shocked plasma. The results - specifically referring to the sources Mkn 421 and BL Lac - are presented in Sect. 3. In Sect. 4 we draw our conclusions.

\section{Internal shocks and radiated spectra}

\subsection{Physical scenario and dynamics}

In this work we use the approximate model of an unsteady wind described in detail in S01 and in the following we report only its most relevant assumptions.

We consider a compact source, of typical dimension $R_{0} \sim$ $10^{14} \mathrm{~cm}$, which produces an unstable relativistic wind characterized by an average luminosity $L_{\mathrm{w}}$. The emission from the wind is obtained by adding pulses radiated in a series of internal shocks that occur in the outflow (Daigne \& Mochkovitch 1998; Spada et al. 2000; Guetta et al. 2001).

The wind flow is described as a sequence of $N=t_{\mathrm{w}} / t_{\mathrm{v}}$ shells, where $t_{\mathrm{w}}$ is the overall duration of the wind emission and $t_{\mathrm{v}} \ll t_{\mathrm{W}}$ is the average interval between two consecutive shells. Each shell is characterized by four parameters:

1. the ejection time $t_{j}$, where the subscript $j$ denotes the jth shell;

2. the Lorentz factor $\Gamma_{j}$;

3. the mass $M_{j}$;

4. the width $\Delta_{j}$ as measured in the lab frame.

The Lorentz factors of the shells are randomly extracted from a uniform distribution between $\Gamma_{m}$ and $\Gamma_{M}$. The masses are also randomly extracted from a uniform distribution with an average value $M_{\mathrm{t}} / N$, where the total mass in the wind $M_{\mathrm{t}}$ is normalized to the jet energy budget. The time intervals $t_{j+1}-t_{j}$ are drawn randomly from a uniform distribution with an average value $t_{v}$. The initial shell width $\Delta_{j}$ is of the same order of $R_{0}$ and the initial internal energy $U_{j}$ is negligible (all of the energy is in the form of bulk kinetic energy).

The dynamics of the wind expansion is characterized by a series of two-shell collisions in which the faster shells catch up with the slower ones. The model computes the collision time of two shells and replaces them with a new shell having mass, Lorentz factor, and internal energy given by the conservation laws for inelastic collisions (for details see S01). Before colliding, the shells are assumed cold with constant thickness $\Delta=R_{0}$ but after collision they get heated and thus assumed to expand at the (comoving) sound velocity. Adiabatic losses during the expansion are taken into account but the bulk Lorentz factor is kept constant since the internal energy is always much smaller than the bulk kinetic one. The magnetic field is generated at each collision without keeping memory of any field generated in previous collisions.

In each collision a forward (FS) and a reverse (RS) shock are formed and propagate into the front and the back shell, respectively. The plasma parameters behind each shock are determined by the jump conditions in mildly relativistic shocks, namely continuity in the energy density and velocity across the contact discontinuity separating the two shells (Panaitescu \& Mészáros 1999). We also take into account the compression of the merged shell width due to the propagation of the FS and RS.

The energy released in each shock is assumed to be distributed among protons, electrons and magnetic field with fractions $\epsilon_{\mathrm{p}}, \epsilon_{\mathrm{e}}$ and $\epsilon_{B}$, respectively. The resulting magnetic field strength allows us to evaluate the synchrotron and the inverse Compton emission.

Electrons are assumed to be accelerated at relativistic energies and injected with a power law spectrum, above a (random) Lorentz factor $\gamma_{\mathrm{b}}$. The actual distribution $N(\gamma)$ which we use to calculate the radiated spectrum is specified below. The value of $\gamma_{\mathrm{b}}$ is determined by requiring that $\int N(\gamma) \gamma m_{\mathrm{e}} c^{2} \mathrm{~d} \gamma$ equals the fraction $\epsilon_{\mathrm{e}}$ of the total available internal energy and accounting for the fact that only a fraction, $\zeta_{\mathrm{e}}$, of electrons is effectively accelerated (in other words we assume the presence of also a population of cold electrons, corresponding to a fraction $\left(1-\zeta_{\mathrm{e}}\right)$ of the total. $N(\gamma)$ here describes the distribution of the relativistic electrons only). We assume that below $\gamma_{\mathrm{b}}$ electrons are accelerated with smaller efficiency, corresponding to a flatter energy distribution (with respect to the slope above $\gamma_{\mathrm{b}}$ ), assumed to be $\alpha \gamma^{-1}$.

In conclusion, by considering the wind dynamics and shock hydrodynamics, the energy density in non-thermal electrons and in magnetic field and the typical random Lorentz factor $\gamma_{\mathrm{b}}$ can be determined for each collision.

\subsection{Local spectra}

We followed the same basic assumptions of S01, which we briefly recall:

- The emitting zone is homogeneous with comoving volume $V=\pi \psi^{2} R^{2} \Delta R^{\prime}$, where $\psi$ is the half-opening angle of 
a conical jet and $\Delta R^{\prime}$ is the comoving thickness of the shell at the collision time, whose expansion while emitting is neglected for simplicity.

- The relativistic particles, embedded in a tangled magnetic field, have the same energy distribution throughout the region corresponding to the shell-shell interaction. This simplification is justified as we are interested in the "average" spectrum, but does not account for spectral details occurring on a timescale shorter than the light crossing time.

- Even in the case of low power BL Lacs, we consider the presence of soft photons external to the jet (identified with the emission from the broad line region, BLR). The external photon luminosity $L_{\mathrm{ext}}=a L_{\mathrm{disk}}$ is produced within $R_{\mathrm{BLR}}$ and abruptly vanishes beyond, and corresponds to a comoving radiation energy density $U_{\text {ext }}=(17 / 12) a L_{\mathrm{disk}} \Gamma^{2} /\left(4 \pi R_{\mathrm{BLR}}^{2} c\right)($ e.g., Ghisellini \& Madau 1996).

High and low power blazars have different line/photoionizing disk luminosities. Following Kaspi et al. (2000), $R_{\mathrm{BLR}}$ and $L_{\text {disk }}$ are related by $R_{\mathrm{BLR}} \propto L_{\text {disk }}^{b}$ with $b \sim 0.7$. Thus blazars with weaker broad emission lines should have smaller BLR, implying that the first collisions occur preferentially outside $R_{\mathrm{BLR}}$.

- Relativistic electrons are injected with a broken power-law energy distribution, $\propto \gamma^{-1}$ below $\gamma_{\mathrm{b}}$ and $\propto \gamma^{-s}$ between $\gamma_{\mathrm{b}}$ and $\gamma_{\max }$, with a power $L_{\mathrm{e}}$. The equilibrium particle distribution $N(\gamma)$, resulting from the continuous injection and cooling processes, is determined through the following procedure.

Above the (comoving) random Lorentz factor $\gamma_{\text {cool }}$, corresponding to a cooling time comparable with the shock crossing time $t_{\text {cross }}$, the distribution is assumed to have a power law index $p=s+1$. Below $\gamma_{\text {cool }}$ there are two different behaviors:

i) if $\gamma_{\text {cool }}>\gamma_{\mathrm{b}}$, we assume $N(\gamma) \propto \gamma^{-s}$ between $\gamma_{\mathrm{b}}$ and $\gamma_{\mathrm{cool}}$, and $N(\gamma) \propto \gamma^{-1}$ for $\gamma<\gamma_{\mathrm{b}}$

ii) if $\gamma_{\text {cool }}<\gamma_{\mathrm{b}}, N(\gamma) \propto \gamma^{-2}$ between $\gamma_{\text {cool }}$ and $\gamma_{\mathrm{b}}$, and $N(\gamma) \propto \gamma^{-1}$ for $\gamma<\gamma_{\text {cool }}$.

- The normalization of $N(\gamma)$ is determined according to whether particles with $\gamma \sim \gamma_{\mathrm{b}}$ can or cannot cool in the timescale $t_{\text {cross }}$ (fast and slow cooling regime, respectively): Fast cooling regime - if most of $L_{\mathrm{e}}$ is radiated in a timescale $<t_{\text {cross }}$ (i.e. $\gamma_{\mathrm{b}}>\gamma_{\text {cool }}$ ) we apply a luminosity balance condition:

$L_{\mathrm{e}}=V m_{\mathrm{e}} c^{2} \int \dot{\gamma} N(\gamma) \mathrm{d} \gamma$,

where $\dot{\gamma}$ is the radiative cooling rate, including synchrotron, synchrotron self-Compton (SSC) and external Compton (EC) losses. In order to allow for multiple Compton scatterings we a priori calculate how many scattering orders $n_{\mathrm{IC}}$ can take place before the Klein-Nishina regime is reached. By considering $\gamma_{\mathrm{b}}$ as the relevant energy

$n_{\mathrm{IC}}=\frac{\ln \left(\gamma_{\mathrm{b}} / x_{\mathrm{B}}\right)}{\ln \left(4 \gamma_{\mathrm{b}}^{2} / 3\right)}-1$, where $x_{\mathrm{B}} \equiv h v_{\mathrm{B}} /\left(m_{\mathrm{e}} c^{2}\right)$ and $v_{\mathrm{B}}=e B /\left(2 \pi m_{\mathrm{e}} c\right)$ is the (nonrelativistic) Larmor frequency. We define $U_{\mathrm{e}} \equiv L_{\mathrm{e}} /\left(\pi R^{2} c\right)$ and introduce the Comptonization parameter $y$ :

$y \equiv \frac{3}{4} \sigma_{\mathrm{T}} \Delta R^{\prime} \int \gamma^{2} N(\gamma) \mathrm{d} \gamma$.

The equality between the injected and the radiated power in the fast cooling regime translates into an equality between the electron and the radiation energy densities, where the latter comprises the synchrotron and the $n_{\mathrm{IC}}$ inverse Compton orders energy density, i.e.

$U_{\mathrm{e}}=y U_{\mathrm{B}}\left(1+\frac{U_{\mathrm{ext}}}{U_{\mathrm{B}}}+y+y^{2}+\ldots . y^{n_{\mathrm{IC}}}\right)$.

This provides the normalization of $N(\gamma)$, assuming the above slopes.

Slow cooling regime - if most of the power is not radiated in $t_{\text {cross }}$, the electrons retain their energy and

$E_{\mathrm{e}}=L_{\mathrm{e}} t_{\text {cross }}=m_{\mathrm{e}} c^{2} V \int N(\gamma) \gamma \mathrm{d} \gamma$

Assuming the same distribution for $N(\gamma)$, its normalization can be determined.

- The slopes of the $N(\gamma)$ distribution are fixed, but the cooling energy is found by iteration as $\gamma_{\mathrm{cool}}$ depends on the amount of SSC radiation, which in turn depends on the exact shape of $N(\gamma)$ (because of Klein-Nishina effects). Since $\gamma_{\text {cool }}$ determines the normalization of $N(\gamma)$, we re-iterate the procedure described above until it converges (usually 3-4 iterations are enough).

- The synchrotron-self-absorption process, the inverse Compton emission and the beaming of the radiation are treated as in S01.

\subsection{Observed spectrum}

Both the particle and radiation local spectra thus determined are not steady, but roughly correspond to those attained after a time $t_{\text {cross }}$ after the shell-shell interaction, at the maximum bolometric radiative output. This spectrum - adopted for the entire duration of the emission - has a normalization modulated as a function of time.

Each photon pulse - with its corresponding spectrum starts at the time of a collision and lasts for a duration set by the combination of the radiative, shock crossing and angular spread timescales. The shape of the pulses (raise and decay in the light curves) is assumed symmetric and linear with time if its duration is determined by geometrical effects and/or by the shock crossing time, as generally occurs for collisions inside the BLR (as typical of powerful blazar like 3C 279). If the pulse duration is instead set by cooling, the pulse still rises linearly, but decays exponentially $\left[\propto \exp \left(-t / 3 t_{\text {cool }}\left(\gamma_{\mathrm{b}}\right)\right)\right]$, implying that the rise and decay times can be different. This usually occurs in weaker blazars, like Mkn 421. The pulses of intermediate BL Lac objects can be characterized by either of these pulse shapes, depending on the broad lines intensity and thus on whether collisions take place within $R_{\mathrm{BLR}}$. 


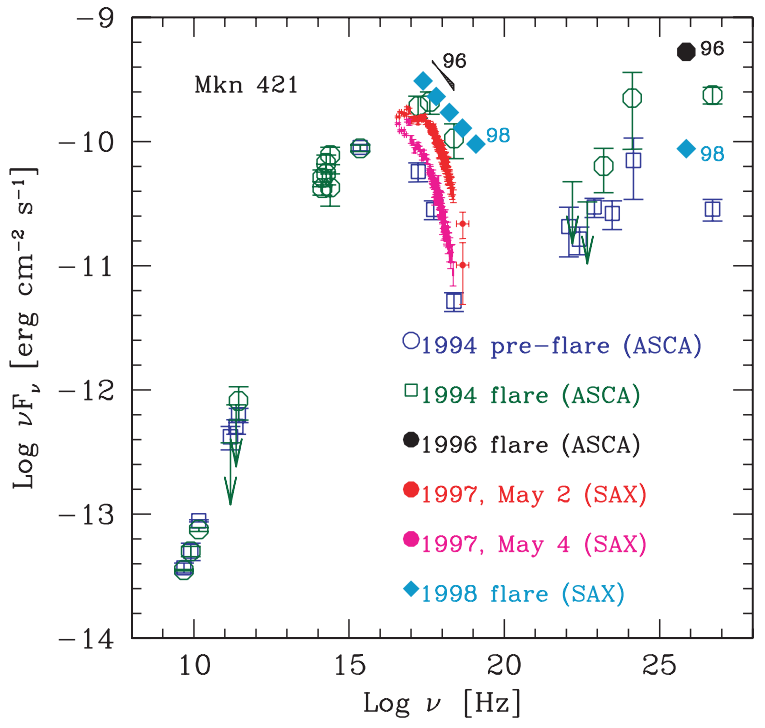

Fig. 1. The SED of Mkn 421 during different observational campaigns, as labeled. For the data points see Costamante \& Ghisellini (2002) and references therein.

The observed spectrum is determined by all of the photons reaching the observer simultaneously: since these are produced at different distances, their different light propagations times should be taken into account. In other words, the resulting spectrum is obtained as the convolution of the emission produced by shocks simultaneously active in the observer frame, taking into account the radius at which collisions occur and the photon travel path.

\section{Results}

The main aim of this work is to explore whether the internal shock scenario can account for the difference of the SED along the blazar sequence. We thus a) concentrate on the global properties, rather than to explain details of the spectrum of a specific object; b) apply the model to Mkn 421 and BL Lac itself which, besides being among the best studied BL Lac objects, are representative of extremely blue and intermediate blazars, respectively, complementing the study already performed for the powerful blazar 3C 279 (S01).

$M k n 421$ - this is one example of bright, extremely blue and strong $\mathrm{TeV}$ emitting blazar. The peaks of its synchrotron and inverse Compton spectra are at $\sim \mathrm{keV}$ and a few hundreds $\mathrm{GeV}$ energies, respectively. The bolometric (observed) power is of order $8 \times 10^{45} \mathrm{erg} \mathrm{s}^{-1}$. The SED of coordinated simultaneous observational campaigns of Mkn 421 are reported in Fig. 1 (Costamante \& Ghisellini 2002).

BL Lac - the prototype of the BL Lacertae class is an intermediate blazars (e.g., Fossati et al. 1998). It is a 1 Jy source, with broad band peaks in the near IR and MeV-GeV band and its total bolometric power is of order $\sim 10^{46} \mathrm{erg} \mathrm{s}^{-1}$. Despite of BL Lac being the prototype of the BL Lac class, it sporadically showed optical emission lines (with maximum observed $E W \sim 6 \AA$; Vermeulen et al. 1995; Corbett et al. 2000). In

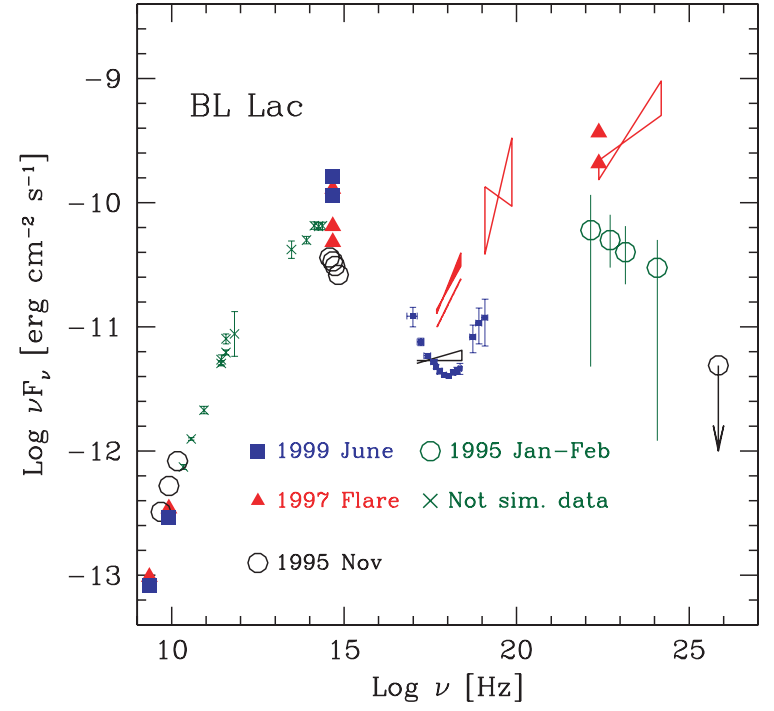

Fig. 2. The SED of BL Lac during different observational campaigns, as labeled. For the data points see Ravasio et al. (2002) and references therein.

July 1997 it underwent a major outburst, followed in the optical and by the CGRO (EGRET), RossiXTE and ASCA satellites. During this flare the entire SED dramatically changed, as illustrated in Fig. 2, with EGRET detecting a flux $(>100 \mathrm{MeV})$ a factor 3.5 times higher than that observed in 1995 (Bloom et al. 1997).

In order to reproduce the SED and variability of these two sources, we took as an initial set of physical parameters that determined for 3C 279 (see S01), and changed as few as possible of them to account for the different broad band SED and "spectral states" of these sources.

It turned out that the global properties of the blazar "sequence" can be reproduced by only changing two key quantities, namely the jet luminosity $L_{\text {jet }}$ and the luminosity $L_{\mathrm{BLR}}$ and extension $R_{\mathrm{BLR}}$ of the external photon field. The other parameters $\left[\epsilon_{B}, \zeta_{\mathrm{e}}\right.$, the slope $n$ of the (high energy) electron distribution, the observing angle $\theta$ ] are only constrained to fit the specific SED. The remaining quantities are at most slightly varied just to optimize the fit. In particular $\epsilon_{\mathrm{e}}$ has been fixed for all sources to 0.5 . In Table 1 we list all of the input parameters adopted to reproduce the time dependent behavior of the simulated sources (for convenience of the reader we also report the same parameters for 3C 279 from S01).

Let us now examine the physical quantities inferred from the modelling and their dependence on $R$.

Efficiency - the radiative efficiency represents the fraction of the dissipated energy at each location channeled into relativistic electrons. In absence of non-radiative losses this energy should be all radiated. As shown in Figs. 3 and 4 for Mkn 421 and BL Lac, respectively, the efficiency decreases simply due to the decrease in the contrast of bulk Lorentz factors of colliding shells. The apparent different efficiencies of BL Lac and 
Table 1. Model input parameters for Mkn 421, BL Lac and 3C 279.

\begin{tabular}{llllllllllll}
\hline \hline Source & $\begin{array}{l}\left\langle L_{\mathrm{jet}}\right\rangle \\
\mathrm{erg} \mathrm{s}^{-1}\end{array}$ & $\Gamma_{m}-\Gamma_{M}$ & $\begin{array}{l}t_{\mathrm{v}} \\
\mathrm{s}\end{array}$ & $\epsilon_{\mathrm{e}}$ & $\epsilon_{B}$ & $\zeta_{\mathrm{e}}$ & $n$ & $\begin{array}{l}L_{\mathrm{BLR}} \\
\mathrm{erg} \mathrm{s}^{-1}\end{array}$ & $\begin{array}{l}R_{\mathrm{BLR}} \\
\mathrm{cm}\end{array}$ & $\begin{array}{l}\theta \\
\text { degree }\end{array}$ \\
\hline Mkn 421 & $8 \mathrm{e} 45$ & $10-20$ & $1.3 \mathrm{e} 4$ & 0.5 & $6 \mathrm{e}-4$ & $8 \mathrm{e}-2$ & 3.1 & $1 \mathrm{e} 42$ & $1 \mathrm{e} 16$ & 2 \\
BL Lac & $4 \mathrm{e} 46$ & $8-20$ & $1.6 \mathrm{e} 4$ & 0.5 & $1 \mathrm{e}-2$ & $1 \mathrm{e}-2$ & 4.3 & $8 \mathrm{e} 42$ & $7 \mathrm{e} 16$ & 4 \\
3C 279 & $1 \mathrm{e} 48$ & $10-25$ & $1.0 \mathrm{e} 4$ & 0.5 & $4 \mathrm{e}-3$ & $4 \mathrm{e}-2$ & 2.1 & $1 \mathrm{e} 45$ & $5 \mathrm{e} 17$ & 2 \\
\hline
\end{tabular}

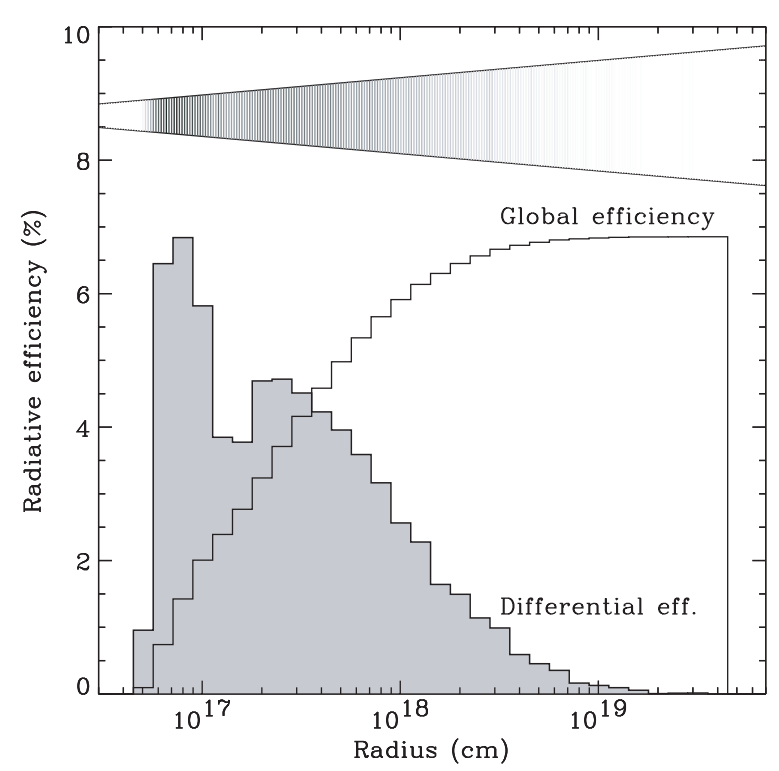

Fig. 3. Radiative efficiency as a function of the collision radius for Mkn 421. The solid line refers to the global efficiency, i.e. the fraction of the total wind kinetic energy $\left(E_{\mathrm{w}}=L_{\mathrm{w}} \times t_{\mathrm{w}}\right)$ radiated on scales smaller than any given radius; the shaded histogram shows the differential efficiency, namely the fraction of $E_{\mathrm{w}}$ radiated for a given radius interval. The cone-like insert in the upper part of the figure shows a grey-tone representation of the differential efficiency of the jet: the darker the color the higher the efficiency.

Mkn 421 reflect only the different viewing angle (chosen to reproduce the detailed properties of the two sources).

Parameters profiles: $\Gamma, B, \gamma_{\mathrm{b}}-$ Figs. 5 and 6 (top three panels) show how the bulk Lorentz factor, the magnetic field, the Lorentz factor $\gamma_{\mathrm{b}}$ of the injected electrons evolve with distance from the nucleus, for Mkn 421 and BL Lac, respectively. Different symbols correspond to shells having experienced a different number of collisions. The bulk Lorentz factors tend toward average values as result of successive collisions and merging of the shells. The range and the asymptotic bulk Lorentz factor $\Gamma$ are fully consistent with the typical values inferred from observations. The magnetic field decreases roughly as $R^{-3 / 2}$ for both sources: it is worth remarking that we assume that a constant fraction $\left(\epsilon_{B}\right)$ of the available energy is converted into magnetic field and no field component survives between successive collisions. The minimum energy of the injected electrons, $\gamma_{\mathrm{b}} m_{\mathrm{e}} c^{2}$, is also a decreasing function of $R$, due to the decrease in the bulk Lorentz factors contrast, implying decreasing efficiency and mean energy per particle.

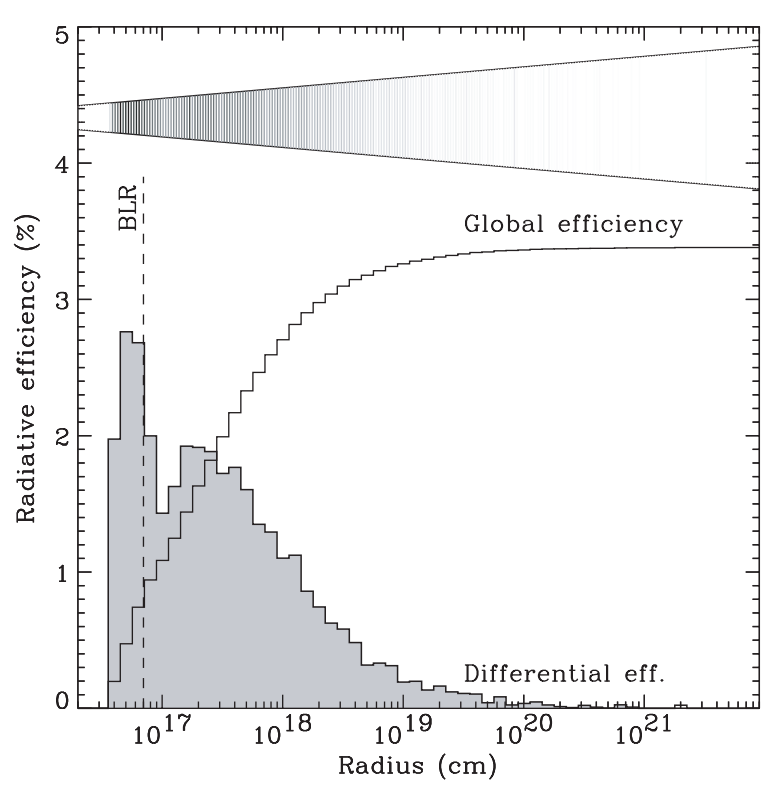

Fig. 4. Radiative efficiency versus the collision radius for BL Lac. The solid line refers to the global efficiency, i.e. the fraction of the total wind kinetic energy $\left(E_{\mathrm{w}}=L_{\mathrm{w}} \times t_{\mathrm{w}}\right)$ radiated on scales smaller than a given radius; the shaded histogram shows instead the differential efficiency, namely the fraction of $E_{\mathrm{w}}$ radiated for a given radius interval. The cone-like insert in the upper part of the figure shows a grey-tone representation of the differential efficiency of the jet: the darker the color the higher the efficiency. The vertical line indicates $R_{\mathrm{BLR}}$.

In general the only difference between Mkn 421 and BL Lac is the smaller spread of the parameters for the former, due to the fact that in Mkn 421 all the collisions occur in the same region.

Parameters profiles: $\gamma_{\text {peak, }}, v_{\text {peak }}-$ the two bottom panels of Figs. 5 and 6 show the spatial profile of the energy of the electrons emitting at the peak of the SED, $m_{\mathrm{e}} c^{2} \gamma_{\text {peak }}$, and the corresponding observed synchrotron peak frequency, $v_{\text {peak }}$.

A significantly different behavior characterizes the two sources. In Mkn $421 \gamma_{\text {peak }}$ is a continuous decreasing function of $R$ and attains large values. This follows from the fact that in low power blazars only high energy electrons can radiatively cool in a finite injection time, thus determining high values of $\gamma_{\text {peak }}$. Specifically, its value is determined by $\gamma_{\max }$ at each collision radius, as set by the spectral fit. The behavior of $v_{\text {peak }}$ simply follows that of $\gamma_{\text {peak }}$ and $B$. On the contrary for BL Lac $\gamma_{\text {peak }}$ (and consequently $\nu_{\text {peak }}$ ) shows a discontinuity. This occurs at $R_{\mathrm{BLR}}$ : inside the BLR electrons are in the fast cooling regime and thus $\gamma_{\text {peak }}=\gamma_{\mathrm{b}}$ while outside the BLR the behavior is similar to that of Mkn 421, namely the 


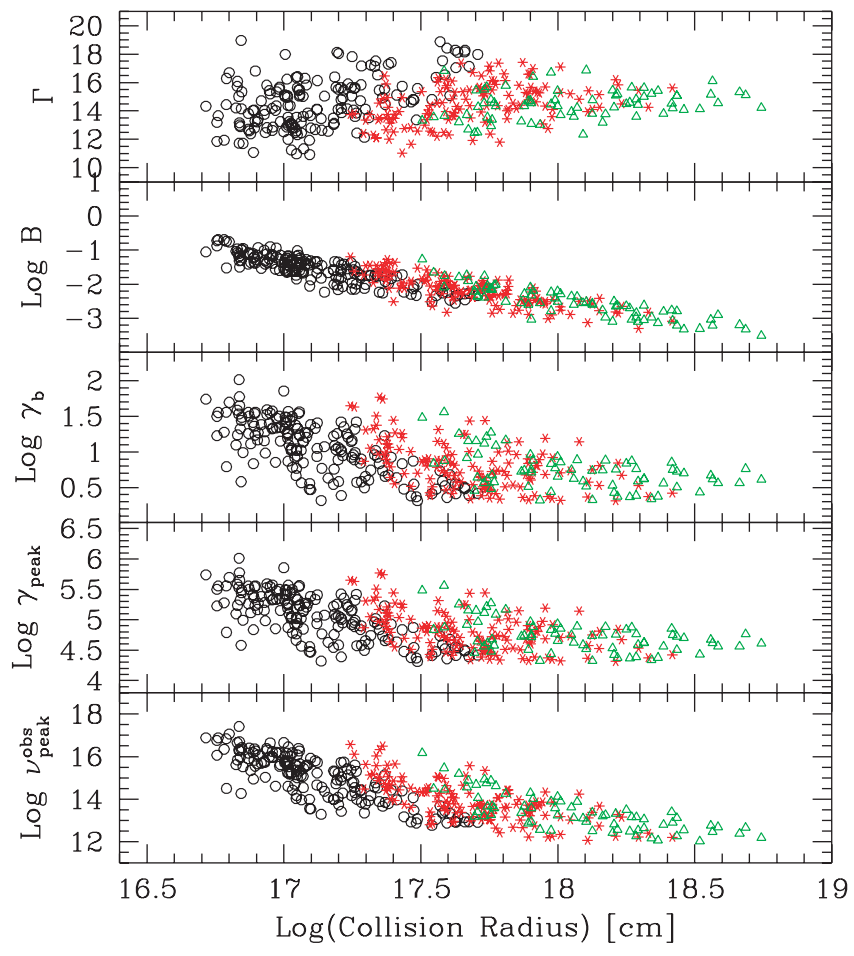

Fig. 5. Evolution of the post-shock shell parameters as a function of the collision radius for Mkn 421. The upper panel shows the bulk Lorentz factor of the merged shell after each collision; the second panel represents the value of the generated magnetic field $\left(\epsilon_{B}=\right.$ $\left.6 \times 10^{-4}\right)$. The third panel shows the minimum random Lorentz factor of the injected electrons $\gamma_{\mathrm{b}}$ (for $\epsilon_{\mathrm{e}}=0.5 ; \zeta_{\mathrm{e}}=0.08$ ). In the fourth and last panels $\gamma_{\text {peak }}$ and $v_{\text {peak }}^{\text {obs }}$ are plotted. Only one tenth of the points is displayed for clarity. Circles refer to collisions in which none of the shells has ever collided before, stars to collisions in which one of the shells has collided, while triangles indicate collisions between shells that have both collided before.

radiative cooling time exceeds the shock crossing time, implying $\gamma_{\text {peak }}=\gamma_{\max }$. Note that for 3C 279 the BLR is even larger and $\gamma_{\text {peak }}=\gamma_{\mathrm{b}}$ for almost all of the collisions.

Spectra at various $R$ - in order to understand how and where the overall spectrum is generated, the spectra produced at different radii are shown in Figs. 7 and 8: in the top panel the average "instantaneous" spectrum of a single shell at $R$ is shown while in the bottom panel the spectra are integrated for all of the shells emitting at a certain time (the flux is thus weighted for the duration of the emission in each shell at a given frequency). While the single shell emission is qualitatively similar for the two sources, the integrated spectra of BL Lac do not simply reproduce the single shell behavior. This is due to the different (shorter) duration of each single shell emission, due to the fast cooling of the electrons (caused by the external Compton). The end result is that in Mkn 421 most of the emission is produced in the inner jet, while in BL Lac the inner part only dominates at the highest frequencies of the two spectral peaks, with interesting consequences on the correlations between variability in different bands.

It is also worth commenting on the extreme differences in the SED of BL Lac at different epochs (as shown in Fig. 2).

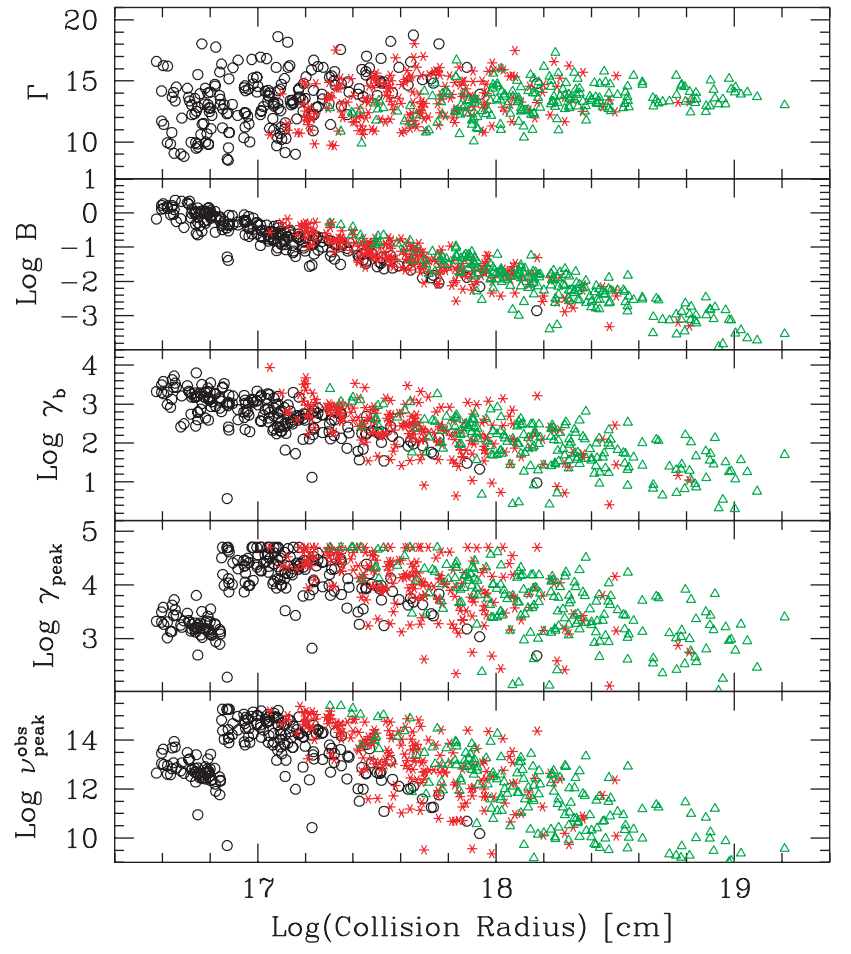

Fig. 6. Evolution of the post-shock shell parameters as a function of the collision radius for BL Lac. The upper panel shows the bulk Lorentz factor of the merged shell after each collision; the second panel represents the value of the generated magnetic field $\left(\epsilon_{B}=10^{-2}\right)$. The third panel shows the minimum random Lorentz factor of the injected electrons $\gamma_{\mathrm{b}}$ (for $\epsilon_{\mathrm{e}}=0.5 ; \zeta_{\mathrm{e}}=10^{-2}$ ). In the fourth and last panels $\gamma_{\text {peak }}$ and $\gamma_{\text {peak }}^{\text {obs }}$ are plotted. Only one tenth of the points is displayed for clarity. Circles refer to collisions in which none of the shells has ever collided before, stars to collisions in which one of the shells has collided, while triangles indicate collisions between shells that have both collided before.

This has been already interpreted as due to variable broad emission lines in the spectrum (mainly $\mathrm{H}_{\alpha}$ and $\mathrm{H}_{\beta}$ ), which would cause a variable contribution of seed photons for the external Compton process. The maximum observed total luminosity from the BLR is $5-8 \times 10^{42} \mathrm{erg} \mathrm{s}^{-1}$ (see e.g. Vermulen et al., for $H_{0}=65 \mathrm{~km} \mathrm{~s}^{-1} \mathrm{Mpc}^{-1}$ ). If one adopts the correlation (Kaspi et al. 2000) between $R_{\mathrm{BLR}}$ and the ionizing continuum luminosity (here assumed to be $\sim 10$ times $L_{\mathrm{BLR}}$ ), $R_{\mathrm{BLR}} \sim$ $5-7 \times 10^{16} \mathrm{~cm}$. This inferred distance is indeed similar to the typical distance of the first shell-shell collisions, suggesting that the collisions sometimes occurs within $R_{\mathrm{BLR}}$, sometimes outside it (see also Ravasio et al. 2002, 2003). Dramatic spectral changes, such as those between the 1995 and the 1997 SED (see Fig. 2) can be therefore accounted for in this scenario.

Light curves - the simulations performed aimed at reproducing (and successfully did) all of the flux range of the SED observed in the various multiwavelength campaigns. However the model also predicts the detailed variability behavior at all frequencies (see also Tanihata et al. 2003) which has not been considered a priori to constrain the model. Let us then examine the light curves in different bands, reported in Figs. 9 and 10 for 

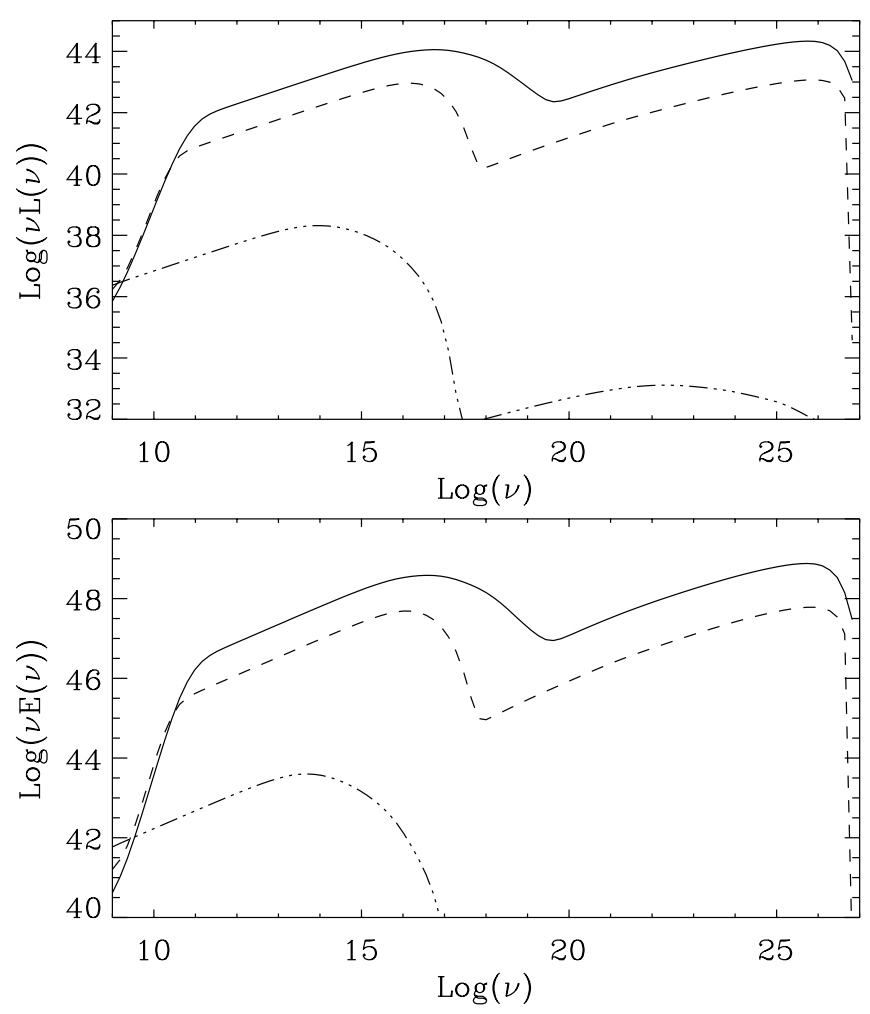

Fig. 7. Average spectra of collisions for Mkn 421. The upper panel shows the average spectrum of the shells without taking into account the duration of the emission. In the lower panel, instead, the duration of the emission of each shell has been taken into account.
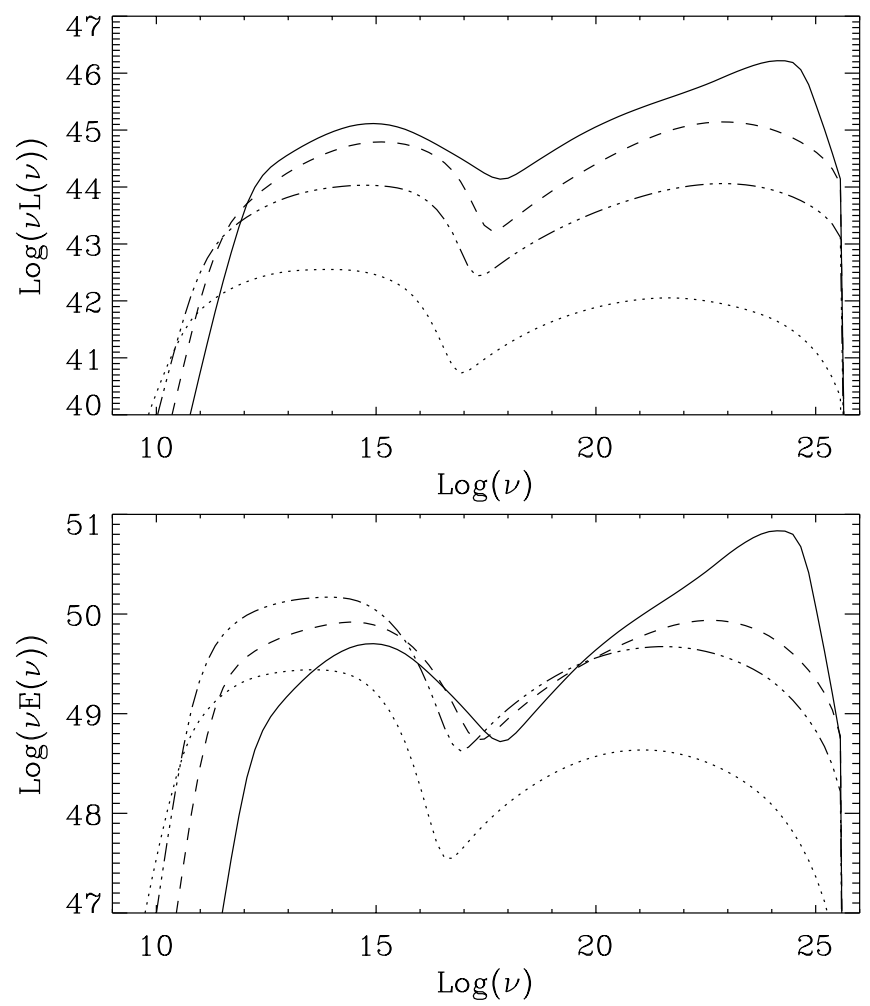

Fig. 8. Average spectra of collisions for BL Lac. The upper panel shows the average spectrum of the shells without taking into account the duration of the emission. In the lower panel, instead, the duration of the emission of each shell has been taken into account.

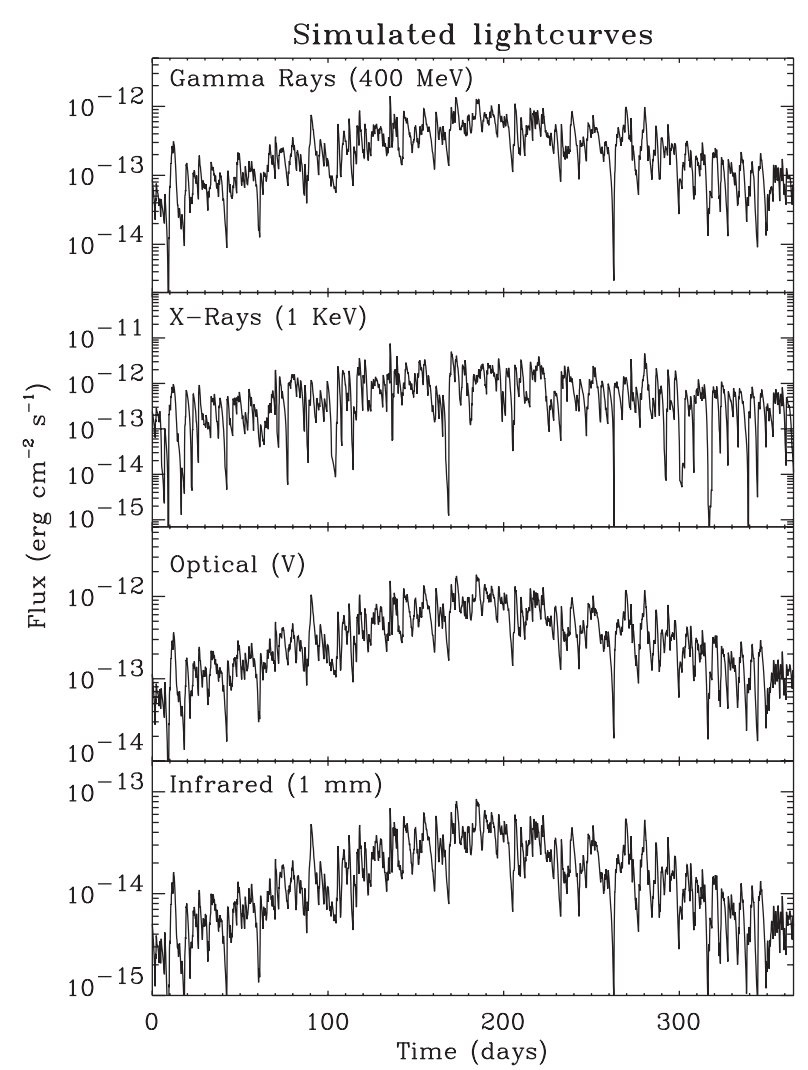

Fig. 9. Simulated light curves for Mkn 421 in different bands, as labeled. A sinusoidal $\left(\propto \sin ^{2}\right)$ modulation with a timescale of $\sim 300$ days has been added in the mass distribution of the shells.

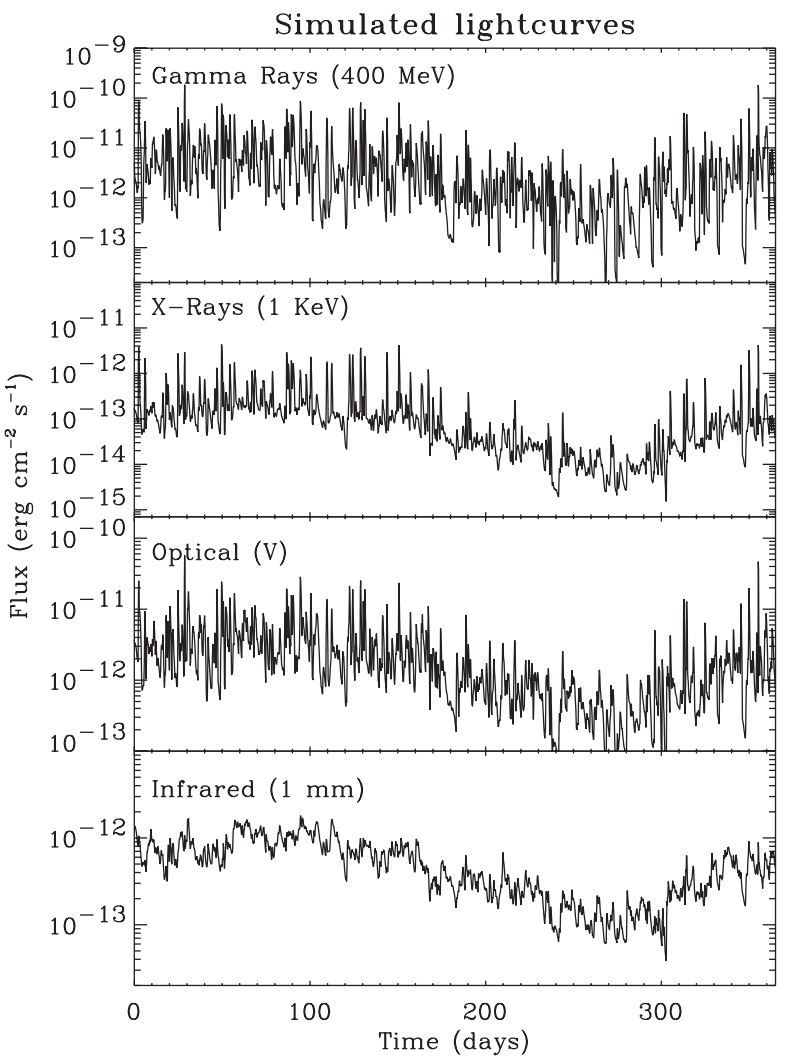

Fig. 10. Simulated light curves for BL Lac in different bands, as labeled. A sinusoidal $\left(\propto \sin ^{2}\right)$ modulation with a timescale of $\sim 300$ days has been added in the mass distribution of the shells. 


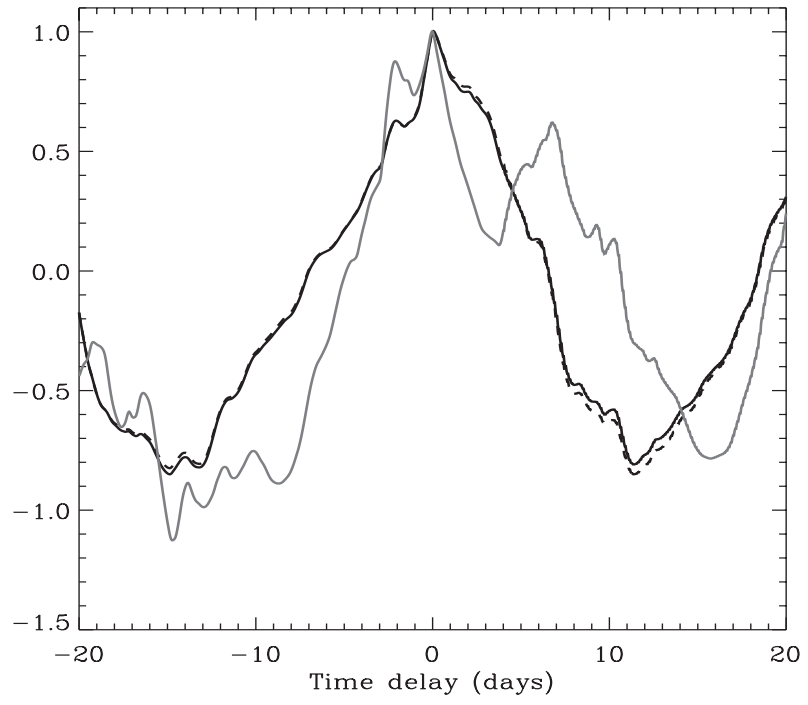

Fig. 11. Results of the cross correlations analysis for Mkn 421, between the $\gamma$-ray and X-ray (solid grey line), optical (solid dark line) and infrared (dashed line) light curves. There are no delays between the different frequencies.

the two sources, and simply compare their variability properties with observations. A movie showing the spectral variability during the whole simulation for Mkn 421 (and 3C 279) can be found at the URL http://ares.merate.mi .astro.it/ $\sim$ gabriele/421/index.html. The long term "periodicity" that appears in the light curves is due to the sinusoidal modulation chosen for the mass distributions of the shells, with a timescale of $\sim 300$ days on the basis of the activity cycles detected in the monitoring of Mkn 501 in the X-ray band (e.g. Fig. 1 in Ghisellini 1998).

In order to assess the potential of the model to reproduce the data, we focused on comparing the simulated light curves with the observed ones, by calculating basic indicators, namely the rms, the cumulative distribution of the variance [(Flux $\langle$ Flux $\left.\rangle)^{2}\right]$, and the minimum variability doubling timescales, defined as $\langle$ Flux $\rangle \Delta t /(\Delta$ Flux $)$. In particular we focused on the $\mathrm{X}$-ray and optical bands for Mkn 421. For the optical variability we sampled the simulated curves with a frequency of about once a day for all the 300 days covered by the simulation and compared the results with the photometric light curves in the $V$ band presented by Tosti et al. (1998). For the X-rays, we considered the results presented by Tanihata et al. (2001) on 6 day continuous observations of the source with ASCA and binned the observed $0.2-2 \mathrm{keV}$ light curve over the same time bins ( 0.125 days) of the simulations.

In the optical band the simulated light curves show significantly larger variability than the observed one (as from Tosti et al. 1998): the rms from the observations is about 4 times smaller than that derived from the model, and the Kolmogorov-Smirnoff test (KS) on the variance distributions rejects the null hypothesis. On the contrary, the comparison reveals promising results for the variability in the X-ray band. Here, the observed and simulated rms are comparable ( 0.2 vs. 0.3 ) and the KS test shows that the variance distributions are marginally compatible (5 per cent level). The X-ray

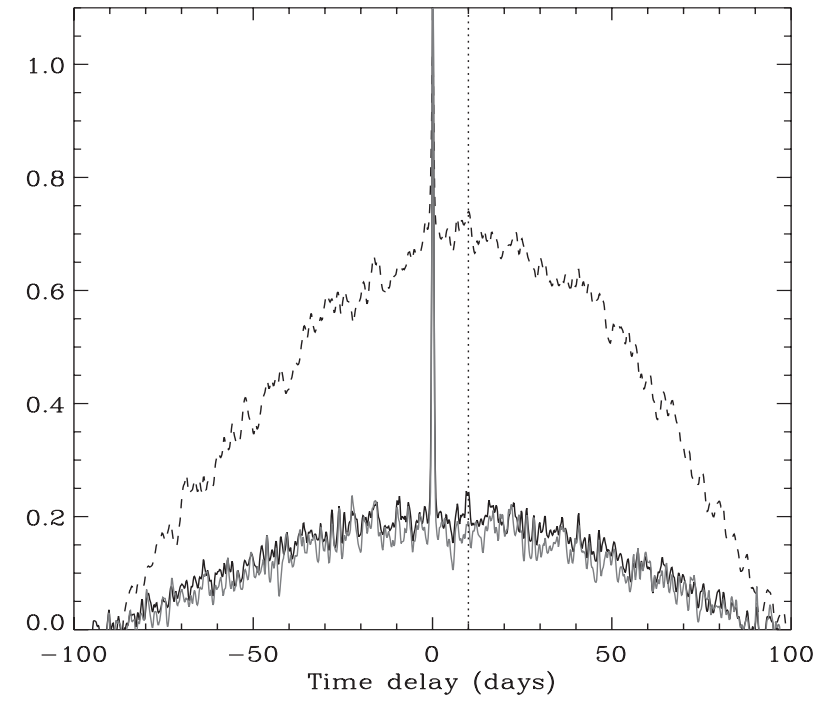

Fig. 12. Results of the cross correlations analysis for BL Lac, between the $\gamma$-ray and X-ray (solid grey line), optical (solid dark line) and infrared (dashed line) light curves. There is a delay between the $\gamma$-ray and the infrared emission of roughly 10 days.

variability properties of the simulated and observed light curves appear to be similar also in terms of the variability timescale, which is of the order of 0.3-0.4 day.

While a proper comparison of the variability properties would require a more sophisticated analysis and better sampled data, these tentative results show that the variability in the $\mathrm{X}$-rays is reasonably reproduced by the model. A significant discrepancy appears instead in the optical, where the model predicts larger variability: this could be in principle improved by modifying the model parameters (e.g. reducing the Lorentz factor contrast and modifying its distribution), although beyond the aim of this paper. We however notice that in the case of Mkn 421 the possible role of the host galaxy emission in reducing the optical variability amplitude should be assessed.

Finally, we computed the cross-correlations between the variability in different energy bands. The results are presented in Figs. 11 and 12 for Mkn 421 and BL Lac, respectively. As discussed above for Mkn $421 \gamma_{\text {peak }}$ is determined, at all radii, by $\gamma_{\max }$, accounting for the absence of lags between the different frequencies (Fig. 11). On the contrary, in BL Lac lags ( $\sim 10$ days) are present between the infrared and the $\gamma$-ray band. In fact, in this source the $\gamma$-ray emission is dominated by the external Compton within $R_{\mathrm{BLR}}$, while the infrared one by synchrotron radiation from collisions occurring outside the broad line region. We remind that in the case of 3C 279 (S01) the infrared emission is also determined by collisions outside $R_{\mathrm{BLR}}$, resulting in lags between the IR and the $\gamma$-rays of $\sim 40$ days.

\section{Discussion}

In this work we have considered internal shocks as the dissipation mechanism responsible for the emission in blazars. As mentioned in the Introduction, this scenario is currently the most accredited to explain the prompt gamma-ray emission in Gamma-Ray Bursts, despite of an efficiency problem (Lazzati et al. 1999). In blazars instead the relatively low efficiency is 
in fact required as, at least in powerful objects, most of the jet power is carried to the large scales.

In S01 it was found that the internal shock model was successful in reproducing the observed SED and variability properties of a powerful blazar, namely 3C 279. However, the SED of this object does not represent the whole of the blazar class, which covers a wide range of spectral characteristics (luminosity, frequency of the peaks of the emission and their relative intensity). Interestingly these parameters appear to be correlated and the whole class can be seen as a sequence: the frequency and the ratio of the low vs high energy peak intensity increase with decreasing luminosity (Fossati et al. 1998).

We show here that the internal shock model can satisfactorily account also for the properties of the lower power blazars. The key parameters driving the phenomenological sequence are the jet power (proportional to the radiated one) and the intensity of the broad lines. These parameters in turn regulate the SED shape, as they control the cooling efficiency of the emitting particles. The global radiative efficiency appears instead to be similar for all of the sources examined.

The internal shock scenario determines a characteristic time interval for the injection of relativistic electrons of the order of the dynamical timescale, when the intensity of the spectrum from each collision is maximized. Two regimes are relevant: fast and slow cooling, corresponding to whether electrons of energy $\gamma_{\mathrm{b}}$ can or cannot radiatively cool in the dynamical time. In highly powerful blazars the fast cooling regime dominates in the inner regions (within the BLR) where also most of the power is dissipated. Consequently the peak frequencies are produced by electrons of energy $\gamma_{\mathrm{b}} m_{\mathrm{e}} c^{2}$. In the weakest blazars instead the slow cooling regime prevails over the whole jet. Consequently only the highest energy electrons can cool over such timescale: the peak frequencies thus shift to high values.

Between these two extremes there are intermediate sources, like BL Lac, with broad lines of intermediate intensity, produced at a distance within which a few shell-shell collisions can occasionally take place. Observationally this corresponds to SED with moderate Compton to synchrotron luminosity ratio, as the scattered seed photons are only the synchrotron ones (in collisions outside the BLR). The rare collisions within the BLR give rise to dramatic changes in the SED characterized by a large increase of the Compton component (e.g. BL Lac itself, see Fig. 2).

The model considered here, which considers selfconsistently the dynamics and spectral emission, predicts also the time dependent spectral properties. In general, the selected parameters allow to reproduce the full range of spectral "states" observed in both BL Lac and Mkn 421. A more detailed comparison performed for Mkn 421, and based on the rms and variability timescales, shows good agreement for the X-ray variability properties, while the simulated optical variations appear to be too large with respect to the considered observed light curve.

An analysis of the predicted cross-correlated variability between the $\gamma$-ray and other bands reveals that only for BL Lac lags ( $\sim 10$ days) are expected between the $\gamma$-rays and the infrared emission.
We conclude that the internal shock scenario can account for the the main properties of blazars and the "blazar sequence".

While it has been previously pointed out that the key quantity in reproducing the different characteristics of blazars is the ratio between the jet and the disc (i.e. broad lines) luminosities, the internal shock scenario discussed here provides more physical insights, namely i) directly connects the radiated jet luminosity with the jet effective power and ii) accounts for the "preferred" distance where most of the luminosity is dissipated. While the latter is similar for high and low power blazars, the BLR is instead located at different distances in the different sub-classes of objects (as determined by the ionizing luminosity).

From a more theoretical point of view, the model does not (yet) address the role of a seed magnetic field amplified by the shell-shell collisions. This would probably lead to a faster synchrotron cooling on the large scales and a flatter dependence of the $B$ field from $R$ (Fig. 3). We intend to further explore this issue. This would also have consequences on the observed polarization in the radio and optical bands (which can be very high in some blazars, e.g. reaching levels of $20 \%$ in the optical, Impey et al. 1991). Furthermore, only internal shocks have been considered in this scenario. It is conceivable that at large jet scales some entrainment may occur causing an interaction of the jet with the external medium, possibly leading to external shocks. As a result the radiative efficiency and large scales synchrotron and inverse Compton emission could be enhanced. Clearly, the inverse Compton emission could be also enhanced if a significant photons field is present externally to the jet even on large scales (such as microwave background and/or beamed nuclear radiation and/or dust emission, e.g. Celotti et al. 2001; Sikora et al. 2002).

Finally, our approximation on the extension of the BLR and its photon density profile vanishing beyond $R_{\mathrm{BLR}}$ tends to underestimate the inverse Compton emission of those internal shocks occurring just beyond $R_{\mathrm{BLR}}$. In this sense the value of $R_{\mathrm{BLR}}$ derived here could be considered as an "effective" distance, slighty different (by a factor $~ 2$ ) from the "real" extension of the BLR photon field which could contribute to the EC process.

Observationally the improved detection sensitivity of the planned TeV Cherenkov telescopes - such as VERITAS, HESS, MAGIC - will allow in the near future to measure emission from BL Lacs in low luminosity states, and thus to estimate their flare activity duty cycles. Since in the internal shock scenario the $\mathrm{TeV}$ emission is largely produced by the few powerful internal collisions, it will be then possible to further constrain model parameters such as the range of bulk Lorentz factors and the initial separation of the shells. Analogous results will be likely achieved for the more powerful blazars in the $\mathrm{GeV}$ band thanks to the AGILE and GLAST satellites.

Acknowledgements. D.G. thanks the Observatory of Brera in Merate for kind hospitality and acknowledges the NSF grant AST-0307502 for financial support. A.C. thanks the JILA fellows, University of Colorado, for the warm hospitality and the Italian MIUR and INAF for financial support. 


\section{References}

Beloborodov, A. M. 2000, ApJ, 539, L25

Bloom, S. D., Bertsch, D. L., Hartman, R. C., et al. 1997, ApJ, 490, L145

Celotti, A., Ghisellini, G., \& Chiaberge, M. 2001, MNRAS, 321, L1

Corbett, E. A., Robinson, A., Axon, D. J., \& Hough, J. H. 2000, MNRAS, 311, 485

Costamante, L., \& Ghisellini, G. 2002, A\&A, 384, 56

Daigne, F., \& Mochkovitch, R. 1998, MNRAS, 296, 275

Fossati, G., Maraschi, L., Celotti, A., Comastri, A., \& Ghisellini, G. 1998, MNRAS, 299, 433

Ghisellini, G., \& Madau, P. 1996, MNRAS, 280, 67

Ghisellini, G., Celotti, A., Fossati, G., Maraschi, L., \& Comastri, A. 1998, MNRAS, 301, 451

Ghisellini, G. 2002, in 25th John Hopkins Workshop [arXiv: astro-ph/0111584]

Ghisellini, 1998, in The active X-ray sky, ed. L. Scarsi, H. Bradt, P. Giommi, \& F. Fiore, Nucl. Phys. B (Elsevier), 397

Guetta, D., Spada, M., \& Waxman, E. 2001, ApJ, 557, 399

Impey, C. D., Lawrence, C. R., \& Tapia, S. 1991, ApJ, 375, 461

Kaspi, S., Smith, P. S., Netzer, H., et al. 2000, ApJ, 533, 631
Lazzati, D., Ghisellini, G., \& Celotti, A. 1999, MNRAS, 309, L13

Padovani, P., Perlman, E. S., Landt, H., Giommi, P., \& Perri, M. 2003, ApJ, 588, 128

Panaitescu, A., \& Mészáros, P. 1999, ApJ, 526, 707

Ravasio, M., Tagliaferri, G., Ghisellini, G., et al. 2002, A\&A, 383, 763

Ravasio, M., Tagliaferri, G., Ghisellini, G., et al. 2003, A\&A, 408, 479

Rees, M. J. 1978, MNRAS, 184, P61

Rees, M. J., \& Mészáros, P. 1994, ApJ, 430, L93

Sikora, M., Blazejowski, M., Moderski, R., \& Madejski, G. M. 2002, ApJ, 577, 78

Sikora, M. 1994, ApJS, 90, 923

Spada, M., Ghisellini, G., Lazzati, D., \& Celotti, A. 2001, MNRAS, 325,1559 (S01)

Spada, M., Panaitescu, A., \& Mészáros, P. 2000, ApJ, 537, 824

Tanihata, C., Takahashi, T., Kataoka, J., \& Madejski, G. M. 2003, ApJ, 584,153

Tanihata, C., Urry, M., Takahashi, T., et al. 2001, ApJ, 563, 569

Tosti, G., Fiorucci, M., Luciani, M., et al. 1998, A\&A, 339, 41

Ulrich, M.-H., Maraschi, L., \& Urry, C. M. 1997, ARA\&A, 35, 445

Wagner, S. J., \& Witzel, A. 1995, ARA\&A, 33, 163

Vermeulen, R. C., Ogle, P. M., Tran, H. D., et al. 1995, ApJ, 452, L5 